\title{
THE SIGNIFICANCE OF HORIZONTAL FORCES IN THE DEVELOPMENT AND CONTROL OF SPINAL DEFORMITIES
}

\author{
By Robert RoAf, M.Ch.Orth., F.R.C.S., F.R.C.S.E. \\ Department of Orthopaedic Surgery, University of Liverpool
}

ONE of the principal aims, if not the principal aim, of any scientific investigation is to describe events in a mathematical form. It is the purpose of this paper to present an approach to the expression in mathematical form of some of the factors, which may cause spinal deformity and to indicate some practical applications which arise from these.

The shape of any object depends on:

I. Its constituents, i.e. the strength, elasticity, plasticity and growth potential of its different materials.

2. The external forces to which it is subjected.

In this paper the discussion is limited to considering the external forces which act on the spine, although it is appreciated that the physical properties and intrinsic growth potential of the spine constituents are also of great importance.

Traditionally there are two possible components of any skeletal deformity which may be combined.

I. Deformities of bone. These may be due to intrinsic factors and/or the result of abnormal external forces such as abnormal muscle action.

2. Deformities of joint. These may be a simple limitation of movement as in the so-called flexion deformity of the hip or knee, or the joint may be in an abnormal anatomical position, e.g. a hyperextended or grossly valgus knee with subluxation. Again the cause may be an intrinsic disorder of the joint, e.g. destruction or irregularity of the articular surfaces, laxity or contracture of the capsule and/or external force such as 'muscle imbalance'.

\section{HORIZONTAL FORCES IN THE GENESIS OF SPINAL DEFORMITY}

In all deformities knowledge of the external forces, their strength, direction and duration is very valuable. Mathematically it is convenient to consider all alterations in shape as deviations from an area of neutral axes, this enables one to express any deformity (angular or rotational) in mathematical terms.

If one wishes to study the forces which are exerted between various skeletal components one should ideally make direct measurements. If, however, the appropriate techniques are not available, one can arrive at a rough estimate of such forces by making either mathematical or mechanical models. Inevitably models introduce an element of oversimplification and must be used with caution. Nevertheless, if direct measurements cannot be made, as for instance on small deep muscles with short tendons, or inside remote inaccessible joints, models can be a useful, if rough, guide to the relative magnitude of the forces exerted, in certain situations under specified conditions. 
It has been customary to regard gravity and longitudinal muscle tension as the two most important mechanical elements in the aetiology of spinal deformity but there are reasons for thinking that horizontal forces-for example rib pressure and the action of the deep transverse or oblique spinal muscles-are often more important.

Any deformity can be considered as an angulation-either linear or rotational. In the models considered in this article, linear angulation is considered primarily: the mathematical analysis of rotational angulation is similar but slightly more complex.

If we consider a square beam subject to a compressing force the formula for the angle $\phi$ to which it is bent is

$$
\tan \phi=\frac{F l}{Y b 4} \sin \theta
$$

where $F$ equals the deforming force, $l$ the length of the beam, $Y$ is Young's modulus, $b$ is the width of the beam, $\theta$ is the initial angle and $\phi$ is the final angle.

The formula for a similar beam subjected to lateral pressure is

$$
\tan \phi=\frac{4 F l^{2}}{Y b 4}
$$

In other words depending on the total length of the beam and the degree of initial deformity, a lateral force will have several times as much deforming action as a longitudinal force, certainly in the initial stages.

An analogous equation for torsion of a cylinder is that the angle equals

$$
\frac{2 L f l}{G r}
$$

where $l$ equals the length of level through which the force $f$ is applied and $G$ equals the modulus of rigidity which in its turn equals Young's modulus divided by $2(l+\sigma)$ where $\Sigma$ is Poisson's ratio, specific for each material,

$$
\text { i.e. } \quad G=\frac{Y}{2(l+\sigma)} \text {. }
$$

All of this indicates clearly that for a given force of a certain magnitude the deformity will be greater the more the force is applied at right angles to the axis of the structure and the greater the lever-that is the greater the distance from the point of movement that the force is applied. This would suggest that in a nearly vertical structure such as a child's spine horizontal forces are especially powerful in creating deformities (in a horizontal structure such as the femoral neck vertical forces are more effective). If one analyses the action of the various spinal muscles treating them as vectors and analysing them into horizontal and vertical components, one can see that acting on the posterior structures there are three groups of forces, the pressures of the ribs on the costo-transverse joints, the pull of the short deep muscles at the spinous processes and the pull of the longitudinal superficial fibres of the erector spinae muscle. Figure I illustrates these forces and their horizontal components. The longitudinal muscles on the concave side will of course only have the action portrayed if they are acting from each end-that is if there are fixed points both cephalic and caudal to the vertebrae concerned. The longitudinal 
muscles on the concave side by increasing the spinal angulation may increase the horizontally displacing component of the body weight vector acting on the body, i.e. the total effect is increased rotation of the vertebra. This state of affairs may

FIG. I

LSM = longitudinal fibres of erector spinae muscle

$\mathrm{OSM}=$ oblique fibres of erector spinae muscle

$\mathrm{CVR}=$ costo-transverse rib pressure on concave side

$\mathrm{CXR}=$ costo-transverse rib pressure on convex side

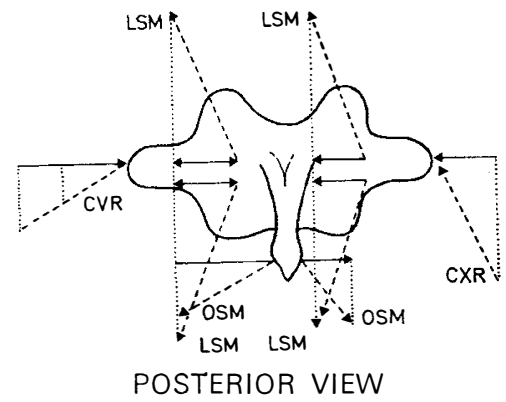

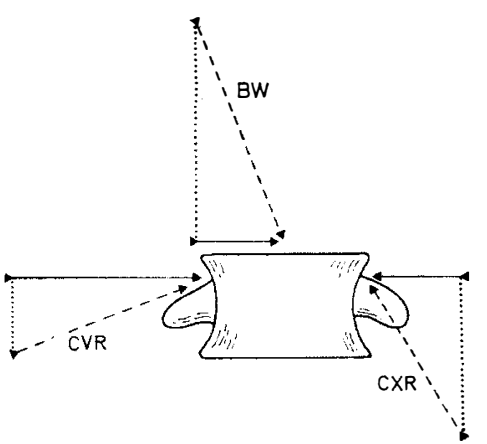

ANTERIOR VIEW
FIG. 3

Some horizontal forces acting on the vertebral body in scoliosis.
Vector analysis of forces acting on the vertebral body and their horizontal components.

$\mathrm{BW}=$ body weight

CVR $=$ costo-vertebral pressure on concave side

$\mathrm{CXR}=$ costo-vertebral pressure on convex side

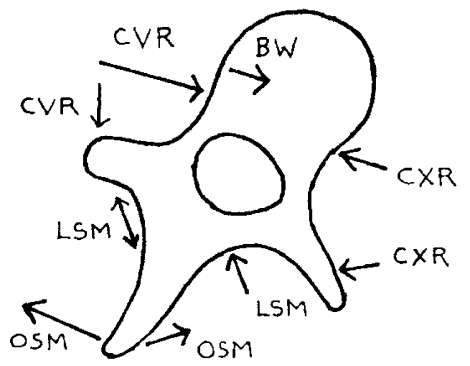

VERTICAL VIEW

occur either by appropriate splinting, external or internal, or by other therapeutic devices. Forces acting on the vertebral body are:

I. The body weight, here again when the upper part of the spine is inclined to the vertical the body weight vector will have a horizontal component.

2. The pressure of the ribs on the costo-vertebral joints.

Again, if as is usually the case, the ribs on the concave side are more horizontal they will have a greater horizontal component (fig. 2). The total horizontal effect of these forces with their appropriate direction and magnitude is shown in Figure 3. 
COMPARISON OF HORIZONTAL (H) VERTICAL(V) AND TENSION(T) FORCES REQUIRED TO STABILISE AN INCLINED BEAM

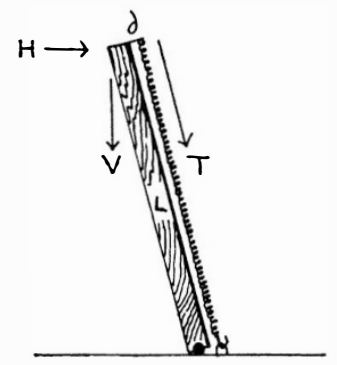

$H L \cos \theta=V L \sin \theta=T \partial$

$\therefore T=\frac{L \cos \theta}{d} H \bumpeq 8 H$

$V=H \cot \theta \Omega 4 H$ for a curve of $30^{\circ}$

ratio is more favourable to horizontal forces for smaller angles

FIG. 4

Comparison of vertical horizontal and longitudinal tension forces required for equilibrium.

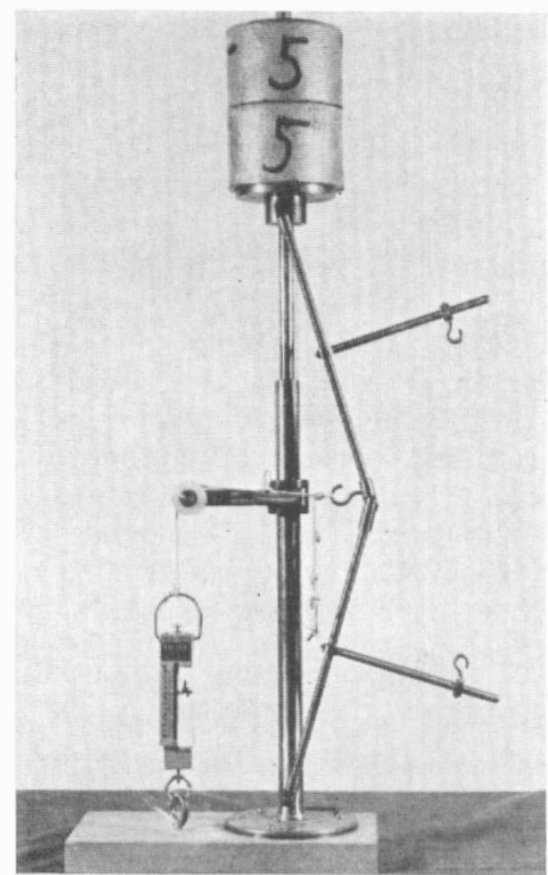

FIG. 5

A horizontal pull of $4 \mathrm{~kg}$. supports the vertical compression load of $\mathrm{I} 0 \mathrm{~kg}$. 
Unfortunately, it is not possible to estimate accurately the force of each of these components but clinical evidence indicates that inequality of the horizontal forces leads to spinal deformity. This is particularly well exemplified in two clinical situations: where a segment of the spine has been fused either by congenital posterior fusion, by the use of instruments, or by anterior fusion. Under these circumstances the deforming action of the muscles is concentrated on the mobile joint above and below the area of fusion and considerable increase in deformity can then occur at these sites. There is also evidence of the overwhelming importance of the erector spinae muscles in a follow-up of patients who have had thoracoplasties. Provided due care is taken to preserve the integrity of the erector spinae muscles it is possible to excise Io ribs without any significant deformity occurring. On the other hand, removal of 3 or 4 ribs with damage to the erector spinae muscles may lead to severe deformity even in mid-adult life (fig. 4).

In a polyarticular model with a slight curvature a small lateral force of $3 \mathrm{~kg}$. doubled the curvature but it required a longitudinal force of $\mathrm{I} 0 \mathrm{~kg}$. to have the same effect. There is, therefore, a considerable prior evidence that horizontal forces are likely to be important in the initiation of spinal deformities (fig. 5).

At this stage it is not possible to state which forces are the most importantthe ribs or the horizontal components of the erector spinae muscles.

As already stated, extensive thoracoplasties can be done without a spinal deformity developing also in a number of acquired neurological conditions, e.g. after severe brain or spinal cord injuries severe spinal deformities often develop even though the action of the intercostal muscles appears still to be symmetrical.

In so far as muscle imbalance is an important factor in progressive spinal deformity and, once a spinal deformity has developed basically normal muscles can act asymmetrically (Roaf). It is obviously important to try to discover which muscles play the most important part.

\section{HORIZONTAL FORCES IN THE CORRECTION OF SPINAL DEFORMITY}

In correcting any joint deformity by either conservative or operative means, one endeavours:

(a) To reduce the resistance of the relevant articulations. The modulus of elasticity of a material is a fixed quantity and cannot be altered but the geometry or amount of material can be changed, e.g. by cutting, stretching or removing some of the material thus reducing the total resistance. In the case of the spine, ribs, ligaments, discs, posterior articulations and vertebral bodies all contribute to the rigidity of the deformity.

(b) To apply a corrective force in such a way that the pressure (force per unit area) is as small as possible.

Mathematically the corrective effect of various forces can be considered by analysing them into horizontal and vertical components. A longitudinal distracting force on the concave aspect has a horizontal component of $2 \sin \frac{\theta}{2}$ and a longitudinal tension force on the convex side has a similar horizontal component (fig. 4).

It is desirable that: 
(a) The force should be applied at the end of the longest possible lever.

(b) The direction of the force should be as near to a right angle to the lever as possible and in the same line as the direction of the deformed element.

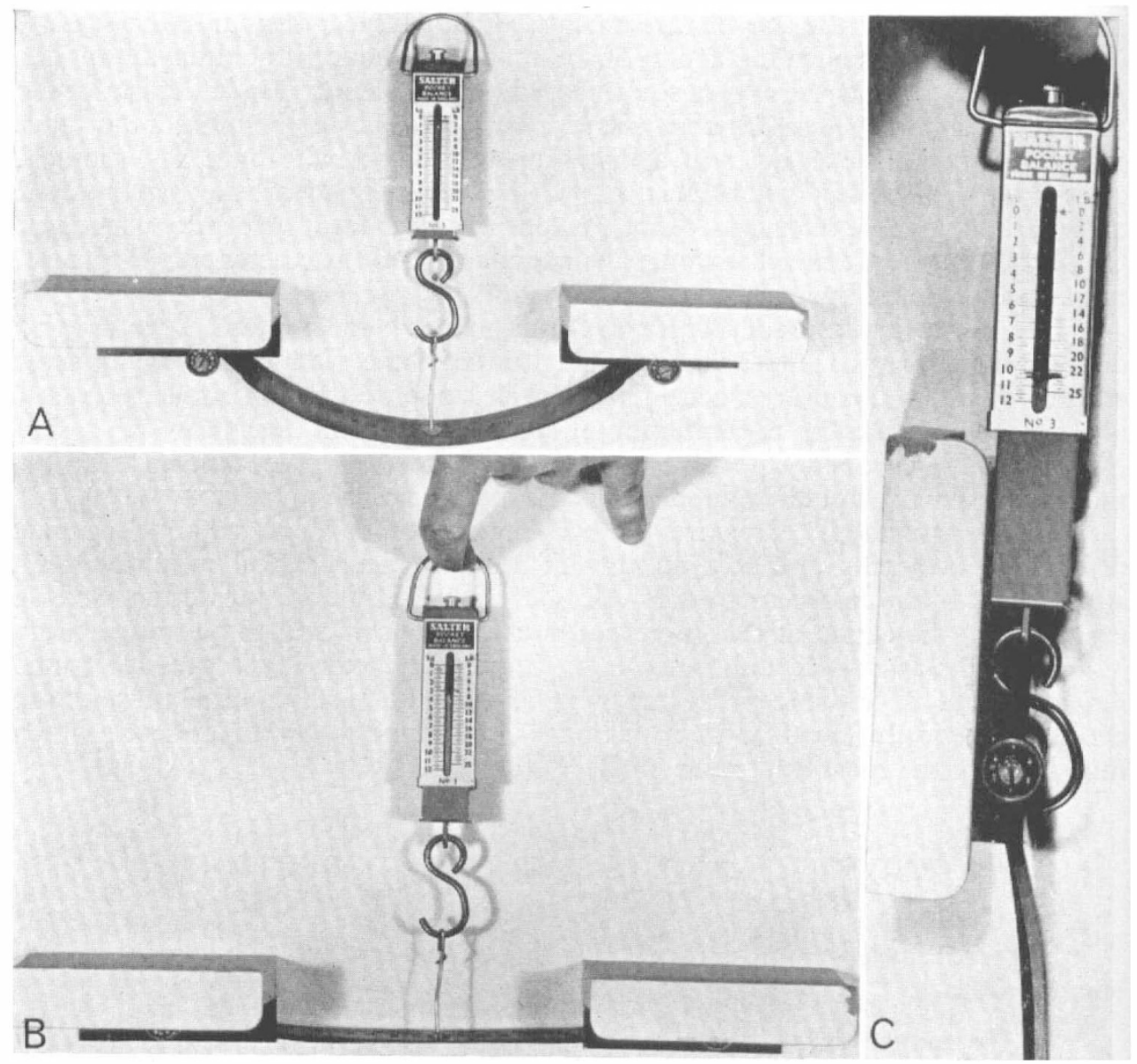

FIG. 6

A, Curved spring $0.5 \mathrm{~kg}$. lateral pull. B, Completely straight with $3 \mathrm{~kg}$. lateral pull. C, Still considerable curvature with Io kg. longitudinal pull.

Corrective forces can be applied by either external or internal means:

I. As direct lateral pressure-that is in a horizontal direction.

2. As a vertically acting extending force on the concave side or as compressing or tensing force on the opposite (convex) side.

A tension wire on the convex side of a curve has a correcting (horizontal force) of $2 \sin \frac{\theta}{2} \times T$, where $T=$ tension and $\theta=$ the angulation of the curve. A longitudinal distraction force also has a horizontal component of $2 \sin \frac{\theta}{2} \times T$. In both cases 
the effective correcting force is much less than the applied force where $\theta$ is under $45^{\circ}$.

The objection might be made that it is too artificial to consider the forces acting on a polyarticular structure as if it was a single angle. When a force is applied to a polyarticular structure such as the spine the ultimate angulation at each joint will be inversely proportional to the resistance of that articulation. In other words each joint will move under the influence of an external force until it reaches its limit at which point it 'locks' and becomes as rigid as the intervening bones. The force is now concentrated at any joint which is still mobile (see fig. 4). Usually when any corrective force is applied to a deformed spine the more mobile joints correct first and often over-correct. One is then left with two nearly straight segments and a relatively localised central deformed portion. In order to correct

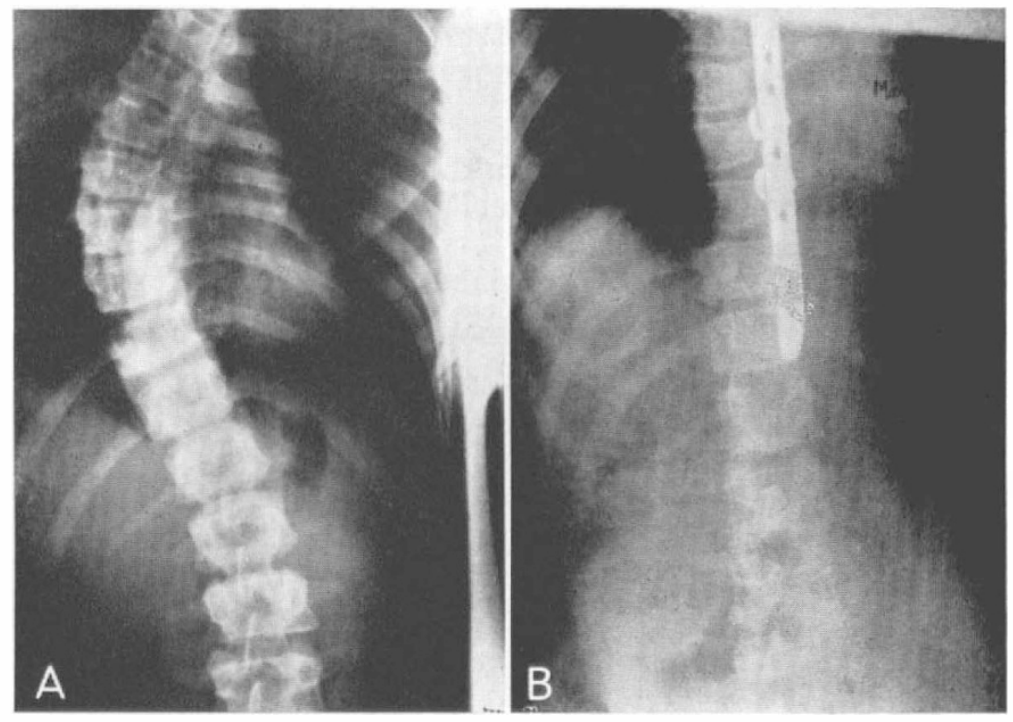

FIG. 7

A, Before correction by horizontal pull. B, After.

this residual deformity the correcting force must be concentrated at this point. Extension or tension forces can only act indirectly and relatively inefficiently on the central residual deformity. Horizontal forces can be applied directly with maximum efficiency.

Figure 6, A, B and c illustrates models in which the relative efficacy of horizontal and vertical corrective forces can be seen.

It is true that the efficacy of longitudinally acting forces can be increased by increasing the length of the levers to which they are attached as the longer the lever the more efficacious is the force, but it is difficult to apply a vertical force (either extensors or tensors) at the end of a long lever as the ribs and transverse processes are unsatisfactory members to which to apply force.

The technical problems of applying corrective horizontal forces to the spine have been considered elsewhere (Roaf, I969) but in general: 
(a) The longer the appliance the better.

(b) The neural arch is the strongest vertebral component to which an internal prosthesis can be attached.

(c) The greater the number of points of attachment and the greater the area over which the force is applied, the less likely it is that one area of bone will be subjected to excessive pressure and undergo necrosis.

(d) The corrective appliance should be designed so as to withstand the deforming forces as directly as possible, i.e. create an opposing force of equal magnitude in the appropriate counter-direction.

Figure 7, A and B shows the results of applying a corrective horizontal force to a scoliotic spine. It is not claimed that this is necessarily the best way of applying a horizontal force, the examples are included to illustrate the efficacy of horizontal correction.

\section{SUMMARY}

Evidence from mathematical and mechanical models and from clinical experience suggests that horizontal forces can be an important cause of spinal angulation. On theoretical grounds a spinal angulation of less than $45^{\circ}$ will be corrected better by a small force which acts horizontally than by a larger force acting vertically. Clinical experience supports this.

\section{ACKNOWLEDGMENT}

I am very grateful to Dr. D. F. Williams, Ph.D., Lecturer in Materials Science and Biomechanics in this department, for advice and criticism.

\section{REFERENCES}

Aвbott, E. G. (I9II). N.Y. Med. M. 93, I2I7.

Allan, F. G. (I955). F. Bone ft Surg. 37B, No. I, 92-Ior.

BICK, E. M. (I948). Source Book of Orthopaedics.

Dwyer, A. F., Newton, N. C. \& Sherwood, A. A. (1969). Clin. Orthop. 62, 192-202.

HARRINGTON, R. (I962). F. Bone ft Surg. 44B, 647.

Kasmin, A. (196I). Ortop. Traumat. Protez. II, II.

Movshovich, I. A. (I960). Ortop. Traumat. Protez. 7, 23.

ROAF, R. (1958). F. Bone ft Surg. 40B, 312.

ROAF, R. (1960). F. Bone $\mathcal{F} t$ Surg. 42B, 40.

ROAF, R. (1963). F. Bone ft Surg. 45B, 637.

ROAF, R. (1969). Proc. Roy. Soc. Med. 62, I47.

ROAF, R. (I970). Paraplegia, 8, 42.

ROAF, R. (I966). Scoliosis, ed. Robert Roaf. Edinburgh and London: E. \& S. Livingstone. 